\title{
Analysis of Artificial Intelligence-based Metaheuristic Algorithm for MPLS Network Optimization
}

\author{
Mohsin Masood ${ }^{1}$, Mohamed Mostafa Fouad ${ }^{2}$, Ivan Glesk ${ }^{1}$, Senior Member, IEEE \\ ${ }^{1}$ Electronics and Electrical Engineering Department, University of Strathclyde, G1 1XW Glasgow, UK \\ ${ }^{2}$ Arab Academy for Science, Technology and Maritime transport, Cairo, Egypt \\ ${ }^{1}$ Electronics and Electrical Engineering Department, University of Strathclyde, G1 1XW Glasgow, UK \\ Tel: (44) 1415482529, email: ivan.glesk@strath.ac.uk
}

\begin{abstract}
Multiprotocol label switched (MPLS) networks were introduced to enhance the network`s service provisioning and optimize its performance using multiple protocols along with label switched based networking technique. With the addition of traffic engineering entity in MPLS domain, there is a massive increase in the networks resource management capability with better quality of services (QoS) provisioning for end users. Routing protocols play an important role in MPLS networks for network traffic management, which uses exact and approximate algorithms. There are number of artificial intelligence-based optimization algorithms which can be used for the optimization of traffic engineering in MPLS networks. The paper presents an optimization model for MPLS networks and proposed dolphin-echolocation algorithm (DEA) for optimal path computation. For Network with different nodes, both algorithms performance has been investigated to study their convergence towards the production of optimal solutions. Furthermore, the DEA algorithm will be compared with the bat algorithm to examine their performance in MPLS network optimization. Various parameters such as mean, minimum /optimal fitness function values and standard deviation.
\end{abstract}

Keywords: Artificial-Intelligence optimization tools, MPLS network optimization, traffic engineering, dolphinecholocation algorithm, bat algorithm.

\section{INTRODUCTION}

Traditional IP networks face serious problems for the integration and service merging of diverse applications that follow numerous traffic characteristics and may require unique quality of services. Whereas, the routing protocols are dependent on old-style routing procedures and thus, fails to secure utilization of resource capability. In the recent years, MPLS has got a noteworthy reputation due to its support for mixed switching techniques, offering various QoS parameters without compromising on network resources. Efficient routing is the key component of traffic engineering management in MPLS networks, which satisfies the QoS with effective resource management. MPLS networks practise multiple protocols and instead of using traditional IP datagram, a short-length labelled packets are used within the network domain for traffic flow and thus, avoiding the complex lookup of routing tables. The edge routers with labels (Label Edge Routers - LER) at MPLS domain are ingress (at source) and egress (at destination side) routers. Whereas, the routers used for traffic forwarding within MPLS domain are label switched routers (LSR). A path is generated between LER (s) for forwarding labelled packets via LSR (s), known as label switched path (LSP). Therefore, the packets flow in the network domain hooked on their labels rather than IP packet headers. Traffic engineering (TE) is considered as the most dominant and impressive object for effective routing of packets in the network. TE is the process of selecting LSP (s) by the data traffic for load balancing on various routers, links switches in the networks. The final goal for using TE is to enable the efficient and consistent network operations synchronized with effective utilization of network resources. Minimizing traffic congestion, MPLS fast reroute of link/ router failures, flexible deployment, class-of-services (CoS) and customer traffic identifications are the number of benefits that's can be achieved in MPLS-TE based networks. However, maximum utilization of links while reducing congestion in the networks are the primary objectives of TE in MPLS networks. Routing protocols plays a vital role for the computation of optimal paths in MPLS-TE entity, where various algorithms can be used for optimal path (s) computation, dependent on objective functions. To compute optimal paths dependent on the objective functions leads to the concept of MPLS network optimization and is considered as NP hard [1], [2]. Recently, Artificial intelligence application-based optimization tools have got momentous appreciation for solving optimization problems in different fields. These optimization tools or techniques are developed on various approaches such as natural, bio, genetic and swarm inspired algorithms [3].

The paper presents the optimization model of MPLS network while considering the computation of optimal paths (based on objective function) using a nature-inspired newly developed dolphin-echolocation algorithm (DEA). In the later sections of the paper, $2^{\text {nd }}$ section formulates the optimization problem with objective function in MPLS networks. Section 3 proposes dolphin-echolocation algorithm (DEA) for MPLS optimization along with pseudo code and, the final segment discusses the results analysis and conclusion. 


\section{PROBLEM FORMULATION OF OPTIMIZATION IN MPLS NETWORK}

MPLS networks are formulated as graphs according to graph theory, where $G=\left\{V_{\text {set }}, E_{\text {set }}\right\}$. Whereas $V_{\text {set }}=$ $\left\{v_{1}, v_{2}, \ldots v_{n}\right\}$ are the number of vertices /nodes /routers and $E_{\text {set }}=\left\{e_{1}, e_{2}, \ldots e_{n}\right\}$ denotes edges /links connected in MPLS networks /graph, respectively. Traffic requests are received at ingress router, where the job for the algorithm is to compute the optimal path dependent on objective function (s) subject to constraints, while the optimal path will be based on optimization model which is described in the following section.

\subsection{Optimization model of MPLS networks}

The optimization model consists of three objective functions, which are based on QoS parameters for MPLS networks such as (a) congestion, (b) routing cost and (c) link utilization.

(a) Congestion

Congestion is considered as one of the main hurdle towards network optimization issue. In MPLS networks, it not only effects the efficiency of an LSP but may produce other network issues such as packets loss/ delay, jitter and end-to-end latency due to overloaded buffer [4]. We adopt the approach that helps to measure the congestion in the network, where the link is congested when its load exceeds to its capacity. Mathematically the congestion model can be described as

$$
\operatorname{minimize}\left\{p_{t}^{l}=\sum_{l=1}^{L_{T}} \rho_{l}\right\}
$$

Where $L_{T}$ is a total number of links in the network, while $\rho_{l}$ is the utilization rate of each link.

(b) Routing cost

Each link $l$ has its own routing cost $C_{m}$ equal to OSPF cost, assigned by network administrator. The path cost $p_{t}^{l}$ is denoted as $c_{t}^{l}$ and thus equal to the sum of its link costs. $x_{t}^{l}$ is an associated bandwidth which is routed on the path [5]. The total routing cost mathematically can be expressed as

$$
\text { minimize }\left\{\sum_{t \in T} \sum_{l=1}^{L_{T}} c_{t}^{l} x_{t}^{l}\right\}
$$

Where $t$ stands for the traffic, meaning a member of total traffic requests $T$, and $L_{T}$ stands for a set of links.

(c) Maximum link utilization:

Maximum link utilization can be achieved by balancing load over each link with its capacity. Each link has its load and capacity, which can be derived as link-load lload over link capacity $l_{c a p}$ [6]. The maximum link utilization over all links is represented by a dependent variable $r$, as an objective function. The goal is a maximum link utilization and can be achieved by minimizing its objective function $r$

$$
\text { minimize }\left\{\max r=\frac{l_{\text {load }}}{l_{\text {cap }}}\right\}
$$

According to our optimization model for MPLS networks, all these mentioned objective functions are combined based on linear combination concept as an objective function that will be minimized to generate the archives of optimal paths for the network, such as

$$
\text { Objective function }=\text { minimize }\left\{\sum_{l=1}^{L_{T}} \rho_{l}+\sum_{t \in T} \sum_{l=1}^{L_{T}} c_{t}^{l} x_{t}^{l}+\max \frac{l_{\text {load }}}{l_{\text {cap }}}\right\}
$$

\section{PROPOSED METHODLOGY}

A recently Dolphin echolocation algorithm (DEA) is the nature inspired algorithm, in which dolphin uses its echolocation characteristics for searching its prey [7]. For the Optimization problem in an MPLS network we used this dolphin echolocation algorithm. In the following section we provide a pseudo code along with mathematically expressions.

\section{Algorithm1. Dolphin echolocation algorithm for MPLS optimization}

Initialize random number of locations $N L$ with variables $N V$, based on the dolphin echolocation property

Create a searching matrix based on $N L \times N V=$ matrix as network (according to graph theory)

Calculate Predefined Probability (PP) loop as follow 


$$
P P\left(\text { loop }_{i}\right)=P P_{1}+\left(1-P P_{1}\right) \frac{\text { loop }_{i}^{\text {power }}-1}{(\text { total number ofloops })^{\text {power }}-1}
$$

Compute the Objective function for each location according to Eq. (4).

Compute the accumulative fitness to dolphin rules as given

For $i=1: \mathrm{NL}$

For $j=1$ : NV

Find the position of location $\left(L_{i, j}\right)$ in the matrix and specify its name as A position

Take range of -Re and Re exploration span

For $k=-\operatorname{Re}$ to $\operatorname{Re}$ end

Evaluate Accumulative Fitness $(\mathrm{AF})=1 / \mathrm{Re} *(\mathrm{Re}-|\mathrm{k}|) *$ minimum fitness function $(\mathrm{i})+\mathrm{A}$

end

Compute the best location using fitness function, call it as "best location" having minimum fitness function

Determine the rest of the variables in Accumulative fitness (AF) as zero.

For $\mathrm{j}$ variables, compute the probability of choosing others by using following expression;

$$
P_{i j}=\frac{A F_{i j}}{\sum_{i=1}^{N L} A F_{i j}}
$$

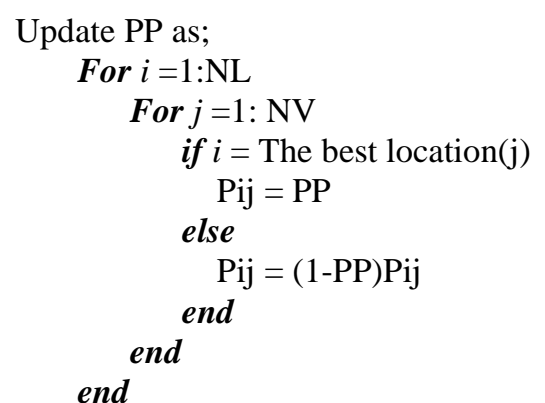

Repeat the algorithm and find the minimum fitness function till the stopping criteria is met.

\section{SIMULATIONS AND RESULTS ANALYSIS}

For implementing the proposed algorithm of MPLS network optimization with a given objective function, we used MATLAB (R2016a) tool. As for the experimental setup, we run algorithm for a different number of nodes per MPLS network such as 10, 20, 30, 50 and 70 nodes. Furthermore, for each network size, we have used different range for -Re and Re, as defined in Eq. (6). For 10 nodes, the range of Re and -Re is -3 to 3, for 20 nodes the range is -5 to 5 . Similarly, for 30,50 , and 70 nodes network, ranges are $[-8,8],[-10,10]$ and $[-13,13]$, respectively. These versions are named as DEA-1, DEA-2, DEA-3, DEA-4 and DEA-5, respectively as shown in Fig. 1. The purpose is to monitor a convergence performance of the algorithm for various network sizes along with various Re and -Re ranges. Fig. 1 depicts the convergence of DEA towards minimum fitness functions as optimal solutions, in which the convergence of various versions of DEA (DEA-1 to DEA-5) are observed. The obtained results from Fig. 1 show that the proposed DEA algorithm successfully generate optimal solutions as paths for different MPLS network sizes. Furthermore, we can see that DEA-1, a 10 nodes network with lowest range of -Re and $\operatorname{Re}[-3,3]$, has a better convergence towards the minimum objective functions and thus produces the best solution after $5^{\text {th }}$ iteration. However, DEA-1 get stuck during $2^{\text {nd }}$ to $3^{\text {rd }}$ iteration of its convergence towards the optimal solution. On the contrary, when we increase the Re and -Re range, as in DEA-2 to DEA-5, the algorithm faces problem of convergence and get stuck during iterations. For example, DEA-4 do not converge and gets stuck between the $3^{\text {rd }}$ and $9^{\text {th }}$ iterations.

For comparative analysis, we compared the presented dolphin echolocation algorithm with the bat algorithm, as a bat algorithm is also an echolocation-based algorithm. As both algorithms are stochastic in nature, we let both algorithms run for 100 times, the data is then collected and measured against parameters such as MEAN, a standard deviation and optimal (minimum fitness function) solution obtained after 100 times run. Our experiments are performed on 20, 50, and 80 nodes networks. Our results in Table 1 show that DEA has achieved far better results in terms of minimum MEAN, the standard deviation and optimal values than the bat algorithm for all networks with any given nodes. Optimal solutions are considered as minimum values. 


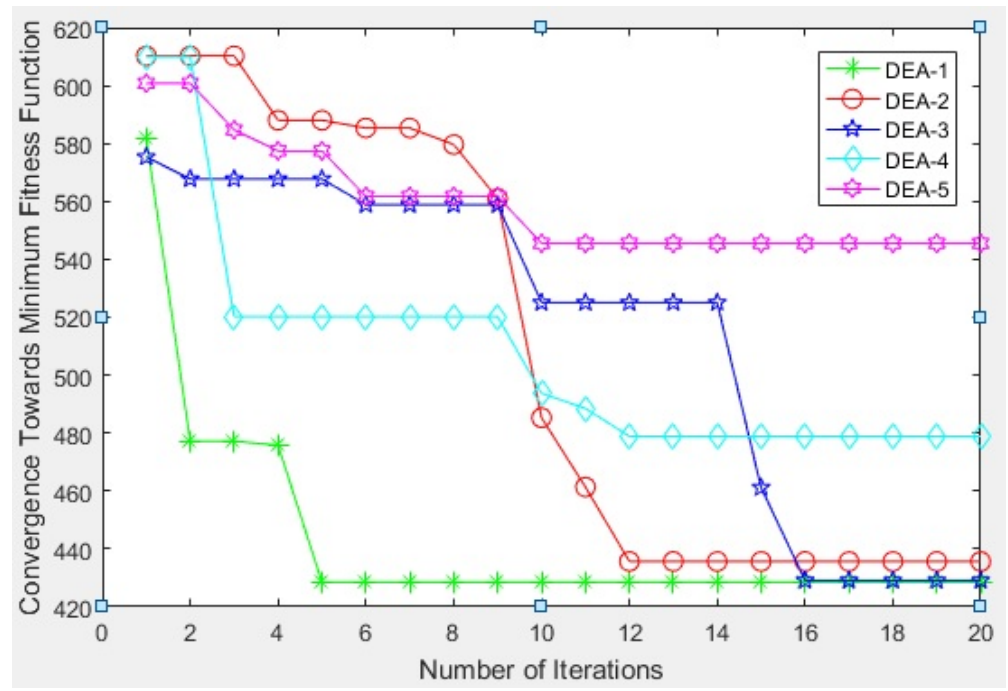

Figure 1. Convergence of Dolphin Echolocation Algorithm (DEA) towards Minimum Fitness function with various DEA versions

Table 1. Comparative study between dolphin echolocation and bat algorithms.

\begin{tabular}{|c|c|c|c|c|c|c|}
\hline \multicolumn{7}{|c|}{ Optimization of MPLS Networks } \\
\hline & \multicolumn{2}{|c|}{20 Nodes } & \multicolumn{2}{c|}{50 Nodes } & \multicolumn{2}{c|}{80 Nodes } \\
\cline { 2 - 7 } & DEA & BAT & DEA & BAT & DEA & BAT \\
\hline Optimal Sol. & 170.23 & 400.34 & 251.90 & 530.01 & 303.10 & 700.12 \\
\hline Mean & 200.66 & 485.45 & 270.67 & 590.18 & 350.05 & 780.16 \\
\hline Std. Dev. & 50.20 & 80.32 & 55.30 & 130.45 & 59.19 & 200.12 \\
\hline
\end{tabular}

\section{CONCLUSION}

The paper has presented an optimization model for MPLS networks having different nodes and investigated a dolphin-echolocation algorithm (DEA) for the MPLS optimization model. From our results shown in Fig. 1 and summarized in Table 1, we can conclude that DEA can be used for the MPLS network optimization for networks having small to large number of nodes. Moreover, this algorithm gives its best performance when using small range of -Re and Re, as the algorithm converge faster and thus provide the optimal solutions with a minimum probability of the algorithm convergence problem. While comparing DEA with the bat algorithm, the results in Table 1 show that DEA outclass the bat algorithm in producing optimal solutions, such as mean and standard deviation parameters.

\section{ACKNOWLEDGEMENTS}

This work was supported by the European Union’s Horizon 2020 Research and Innovation Program through the Marie Sklodowska-Curie under Grant 734331.

\section{REFERENCES}

[1] T. Barabas, D. Ionescu, and S. A. Veres: Traffic engineering algorithm for differentiated multicast services over MPLS networks, in Proc. $7^{\text {th }}$ SACI, Timișoara, Romania, May 2012.

[2] M. Masood, M. Fouad, and I. Glesk: A Pareto based approach with elitist learning strategy for MPLS/ GMPS networks, in Proc. $9^{\text {th }}$ CEEC, Colchester, UK, Sep. 2017.

[3] J. Gero: Artificial intelligence and engineering optimization. Engineering Optimization, vol. 12, pp. 89-90, 1987.

[4] N. El Mawass, A. Sardouk, and S. El Falou: Offline multi-class flow allocation in MPLS networks using a distributed multi-objective genetic algorithm, in Proc.19 ${ }^{\text {th }}$ ICT, Jounieh, Lebanon, Apr. 2012.

[5] M. Masood, M. Fouad, and I. Glesk: Proposing bat inspired heuristic algorithm for the optimization of GMPLS networks, in Proc. 25 $5^{\text {th }}$ TELFOR, Belgrade, Serbia, Nov. 2017.

[6] M. Huerta, X. Hesselbach, and R. Fabregat: An approach to optimizations links utilization in MPLS networks, in Proc. ICCGI'06, Bucharest, Romania, Aug. 2006.

[7] A. Kaveh and N. Farhoudi: A new optimization method: Dolphin echolocation. Advances in Engineering Software, vol. 59, pp. 53-70, 2013. 